\title{
The algebraic approach for the derivation of ladder operators and coherent states for the Goldman and Krivchenkov oscillator by the use of supersymmetric quantum mechanics
}

\author{
Damian Mikulski • Krzysztof Eder • \\ Marcin Molski
}

Received: 10 September 2013 / Accepted: 24 February 2014 / Published online: 1 April 2014 (C) The Author(s) 2014. This article is published with open access at Springerlink.com

\begin{abstract}
The ladder operators for the Goldman and Krivchenkov anharmonic potential have been derived within the algebraic approach. The method is extended to include the rotating oscillator. The coherent states for the Goldman and Krivchenkov oscillator, which are the eigenstates of the annihilation operator and minimize the generalized position-momentum uncertainty relation, are constructed within the framework of supersymmetric quantum mechanics. The constructed ladder operators can be a useful tool in quantum chemistry computations of non-trivial matrix elements. In particular, they can be employed in molecular vibrational-rotational spectroscopy of diatomic molecules to compute transition energies and dipole matrix elements.
\end{abstract}

Keywords Ladder operators · Coherent states - Algebraic methods · Goldman and Krivchenkov potential · Isospectral potential · Darboux transformation · Anharmonic oscillator $\cdot$ Supersymmetry approach

\section{Introduction}

Algebraic construction of coherent states and ladder operators for anharmonic oscillators, described by exactly solvable potentials, is one of the useful tasks to realize in the domain of quantum mechanics. The term coherent reflects the fact that such states are localized on the corresponding classical trajectory and don't change their functional

D. Mikulski $(\bowtie) \cdot$ M. Molski

Department of Theoretical Chemistry, Faculty of Chemistry,

A. Mickiewicz University, ul. Grunwaldzka, 60-780 Poznan, Poland

e-mail: dmkwant@amu.edu.pl

D. Mikulski · K. Eder

Gen. Zamoyska and Helena Modrzejewska High School no.2,

ul. Matejki 8/10, 60-766 Poznan, Poland 
form during propagation. Coherent states can be defined in three ways: (1) by means of a displacement oscillator acting on the ground state of an oscillator, (2) in terms of the eigenstates of an annihilation operator and (3) by the position and momentum of the minimum uncertainty states. It should be pointed out that the definition (1) depends on the analytical form of a displacement operator, which is specific to a harmonic oscillator [1], hence in this case approximate coherent states can be constructed employing, for example, the Nieto and Simmons [2] or Kais and Levine [3] procedures.

The ladder operators can be constructed using the algebraic or supersymmetric (SUSY) approaches [4,5] which have been successfully applied to the Morse, PöschlTeller, radial harmonic and other oscillators [6-15]. The fundamental study in this direction was developed by Schrödinger [16], who introduced the factorization method into quantum mechanics. This concept was generalized by Infeld and Hull [17]. In their paper a detailed analysis of the factorizable oscillators was presented. Recently, Dong et al. derived the raising and lowering operators for the Morse [18], Pöschl-Teller [19] and pseudoharmonic oscillators [20] employing some properties of the associated Laguerre and Legandre polynomials. These operators satisfy the commutation relation for the SU(2) Lie group [21]. The Morse oscillator and others have been studied both in terms of $\mathrm{SO}(2,1)$ and $\mathrm{SU}(2)$ Lie groups. Also Avram and Drặgặnescu [22] have constructed the ladder operators for the Morse potential. They used properties of the confluent hypergeometric function in the recurrent analytic form. Recently, Dong et al. [23] proposed a new anharmonic oscillator in the form:

$$
V(r, \theta)=\frac{1}{2} \mu \omega^{2} r^{2}+\frac{\hbar^{2}}{2 \mu} \frac{\alpha}{r^{2}}+\frac{\hbar^{2}}{2 \mu} \frac{\beta \cos ^{2} \theta}{r^{2} \sin ^{2} \theta}
$$

where $\mu, \omega, \alpha$ and $\beta$ denote the reduced mass of a particle, the angular frequency and two dimensionless parameters, respectively. These authors presented the exact solutions of the Schrödinger equation with this oscillator. Moreover, in this study the ladder operators were constructed directly from the normalized radial function.

Goldman and Krivchenkov introduced a simple anharmonic potential, which can be written in the following form [24]:

$$
V(r)=B r^{2}+\frac{A}{r^{2}}
$$

in which A, B are constants and $\mathrm{r}$ denotes the internuclear separation. The Goldman and Krivchenkov potential (G-K potential) is one of the few for which the Schrödinger equation has an exact analytical solution. This potential is a generalization of the ordinary harmonic oscillator in three dimensions, in which parameter $\mathrm{A}$ is a continuous parameter in the range $[0, \infty]$ instead of the values determined by the rotational quantum number. The $\mathrm{G}-\mathrm{K}$ oscillator is introduced for a description of the bond-stretching vibrations of diatomic molecules. Thus, this potential is a realistic zero-order model useful for description of anharmonic vibrations in diatomic molecules.

The coherent states for the Morse potential have been constructed using different approaches. Perelomov [25] constructed the coherent states using the Lie group symmetry. Employing this approach, Dong [26] obtained the coherent states for the Morse 
potential basing on the SU(2) Lie algebra. Nieto and Simmons [27] proposed a new method for the construction of coherent states of arbitrary oscillators based on the classical equation of motion and applied it to the Morse oscillator. Benedict and Molnar [4] presented an algebraic method employing the SUSY ladder operators to the construction of the coherent states for the one-dimensional Morse oscillator. Moreover, they determined the unitary displacement operator, which generates coherent states from the ground states.

The study of the Barut-Girardello coherent states [28] has attracted much attention. These states can be assumed as eigenstates of the lowering operator of an arbitrary quantum system. Brief [29] considered the Barut-Girardello coherent states using the properties of the Lie algebra SU(1,1). However, it was Fakhri and Chenaghlou [30] who constructed these coherent states for the Morse oscillator as a linear combination of the quantum states corresponding to this potential. A generalization of the BarutGirardello approach was investigated by Gazeau and Klauder [31,32]. Adopting this approach Popov [33] obtained Barut-Girardello coherent states for the Morse potential. Chenaghou and Fakhri [10] derived these coherent states for the Eckart and RosenMorse potentials.

The purpose of the present work is to construct the ladder operators and minimumuncertainty coherent states for the $\mathrm{G}-\mathrm{K}$ oscillator and to generalize the approach to include the rotating $\mathrm{G}-\mathrm{K}$ oscillator. The work is organized as follows. In the second section, we derive the ladder operators from the analytical wave function of the G$\mathrm{K}$ oscillator. In the third part, this method is extended to include the rotating $\mathrm{G}-\mathrm{K}$ oscillator. In the fourth chapter the coherent states and an isospectral potential of the G-K potential have been obtained using the SUSY approach. In the next section, we demonstrate that these coherent states minimize the generalized position-momentum uncertainty relation.

The derived ladder operators for the $\mathrm{G}-\mathrm{K}$ anharmonic and the corresponding $\mathrm{G}-$ $\mathrm{K}$ rotating oscillator can be utilized in rotational-vibrational molecular spectroscopy of small diatomic molecules to compute matrix elements and rotational-vibrational transition probabilities in the electronic states of these molecules.

\section{Ladder operators for the G-K oscillator with zero total angular momentum}

In order to determine the ladder operators for the $\mathrm{G}-\mathrm{K}$ potential we employ the algebraic method. The starting point for the realization of this aim is the vibrational Schrödinger equation with the G-K potential (1.2)

$$
\left(-\frac{d^{2}}{d r^{2}}+B^{\prime} r^{2}+\frac{A^{\prime}}{r^{2}}-\varepsilon_{v}\right) \Psi(r)_{v}=0
$$

in which $A^{\prime}=\frac{2 A m}{\hbar^{2}}, B^{\prime}=\frac{2 B m}{\hbar^{2}}, \varepsilon_{v}=\frac{E_{v} 2 m}{\hbar^{2}}$, and $v=0,1,2,3 \ldots$ is the vibrational quantum number. Equation (2.1) has an exact analytical solution. In particular, the energy spectrum of the Schrödinger equation is given by Hall and Saad [24]

$$
E_{v}=2 \beta(2 v+\gamma)
$$


in which

$$
\beta=\sqrt{B^{\prime}}, \quad \gamma=1+\sqrt{A^{\prime}+1 / 4} .
$$

The normalized solution of Eq. (2.1) takes the form [24]:

$$
\Psi(r)_{v}=(-1)^{v}\left[\frac{2 \beta^{\gamma}(\gamma)_{v}}{v ! \Gamma(\gamma)}\right]^{1 / 2} r^{\gamma-\frac{1}{2}} \exp \left(-\frac{\beta r^{2}}{2}\right) F\left(-v, \gamma, \beta r^{2}\right)
$$

where $F(a, b, x)$ stands for confluent hypergeometric (Kummer) function defined by the Gamma function

$$
F(a, b, x)=\sum_{n=0}^{\infty} \frac{\Gamma(a+n) \Gamma(b) x^{n}}{\Gamma(b+n) \Gamma(a) n !} .
$$

In wave function (2.4) $(\gamma)_{v}$ is the Pochhammer symbol defined by

$$
(\gamma)_{v}=\frac{\Gamma(\gamma+v)}{\Gamma(\gamma)}
$$

The relation between the confluent hypergeometric function and the associated Laguerre polynomials can by written as

$$
L_{n}^{m}(x)=\frac{\Gamma(n+m+1)}{n ! \Gamma(m+1)} F(-n, m+1, x),
$$

in which $L_{n}^{m}(x)$ denotes the associated Laguerre polynomial:

$$
L_{n}^{m}(x)=\sum_{k=0}^{n}(-1)^{k} \frac{[(n+m) !]^{2}}{(n-k) !(m+k) ! k !} x^{k}
$$

Therefore, after changing the variable $x=\beta r^{2}$, we can write the wave function (2.4) in the form

$$
\Psi(x)_{v}=C_{v} x^{\frac{1}{2}\left(\gamma-\frac{1}{2}\right)} \exp \left(-\frac{x}{2}\right) L_{v}^{\gamma-1}(x),
$$

in which

$$
C_{v}=(-1)^{v}\left[\frac{2 \beta^{\gamma}(\gamma)_{v}}{v ! \Gamma(\gamma)}\right]^{1 / 2} \beta^{-\frac{1}{2}\left(\gamma-\frac{1}{2}\right)} \frac{v ! \Gamma(\gamma)}{\Gamma(v+\gamma)} .
$$

The differential ladder operators can be constructed directly from the wave function (2.9). We look for the operators of the following representation:

$$
\hat{K}_{+}=A_{+}(x) \frac{d}{d x}+B_{+}(x) \text { and } \hat{K}_{-}=A_{-}(x) \frac{d}{d x}+B_{-}(x) .
$$

These operators satisfy the following properties:

$$
\hat{K}_{+} \Psi(x)_{v}=K_{+}^{v} \Psi(x)_{v+1} \text { and } \hat{K}_{-} \Psi(x)_{v}=K_{-}^{v} \Psi(x)_{v-1} \text {. }
$$


Then the raising and lowering operators can be constructed by taking advantage of the first derivatives of the associated Laguerre polynomials [34]

$$
\begin{aligned}
& x \frac{d}{d x} L_{n}^{\alpha}(x)=n L_{n}^{\alpha}(x)-(n+\alpha) L_{n-1}^{\alpha}(x), \\
& x \frac{d}{d x} L_{n}^{\alpha}(x)=(n+1) L_{n+1}^{\alpha}(x)-(n+\alpha+1-x) L_{n}^{\alpha}(x) .
\end{aligned}
$$

Employing Eq. (2.13) and the result of the action of the differential operator $\frac{d}{d x}$ on the wave function (2.9) we get

$$
\begin{aligned}
\frac{d}{d x} \Psi(x)_{v}= & \frac{\left(\gamma-\frac{1}{2}\right)}{2 x} \Psi(x)_{v}-\frac{1}{2} \Psi(x)_{v} \\
& +C_{v} x^{\frac{1}{2}\left(\gamma-\frac{1}{2}\right)} \exp \left(-\frac{x}{2}\right) \frac{d}{d x} L_{v}^{\gamma-1}(x) .
\end{aligned}
$$

After simple calculation, we finally obtain the following result:

$$
\begin{aligned}
\frac{d}{d x} \Psi(x)_{v}= & \frac{\gamma-\frac{1}{2}}{2 x} \Psi(x)_{v}-\frac{1}{2} \Psi(x)_{v} \\
& +C_{v} \Phi(x)\left[\frac{v}{x} L_{v}^{\gamma-1}(x)-\frac{v+\gamma-1}{x} L_{v-1}^{\gamma-1}(x)\right],
\end{aligned}
$$

where $\Phi(x)=x^{\frac{1}{2}\left(\gamma-\frac{1}{2}\right)} \exp \left(-\frac{x}{2}\right)$.

Hence, the lowering operator takes the following form:

$$
\hat{K_{-}}=-\left[x \frac{d}{d x}-\frac{1}{2}\left(\gamma-\frac{1}{2}\right)+\frac{1}{2} x-v\right],
$$

whereas its coefficient is given by

$$
K_{-}^{v}=(v+\gamma-1) \frac{C_{v}}{C_{v-1}}
$$

The form of Eq. (2.17) clearly indicates that the lowering operator annihilates the vibrational ground state:

$$
\hat{K}_{-} \Psi(x)_{0}=0 \text {. }
$$

In a similar manner, we construct the raising operator. Using Eq. (2.14) and the result of the action of the differential operator $\frac{d}{d x}$ on the wave function (2.9) we get

$$
\frac{d}{d x} \Psi(x)_{v}=\frac{\gamma-\frac{1}{2}}{2 x} \Psi(x)_{v}-\frac{1}{2} \Psi(x)_{v}+C_{v} x^{\frac{1}{2}\left(\gamma-\frac{1}{2}\right)} \exp \left(-\frac{x}{2}\right) \frac{d}{d x} L_{v}^{\gamma-1}(x)
$$


A simple calculation provides the following result:

$$
\frac{d}{d x} \Psi(x)_{v}=\frac{\gamma-\frac{1}{2}}{2 x} \Psi(x)_{v}-\frac{1}{2} \Psi(x)_{v}+C_{v} \Phi(x)\left[\frac{v+1}{x} L_{v+1}^{\gamma-1}(x)-\frac{v+\gamma-x}{x} L_{v}^{\gamma-1}(x)\right] .
$$

Hence, we obtain the following form of the raising operator:

$$
\hat{K_{+}}=x \frac{d}{d x}-\frac{1}{2}\left(\gamma-\frac{1}{2}\right)-\frac{1}{2} x+v+\gamma
$$

which coefficient is given by

$$
K_{+}^{v}=(v+1) \frac{C_{v}}{C_{v+1}}
$$

\section{Ladder operators for the rotating $\mathrm{G}-\mathrm{K}$ oscillator}

The starting point for the derivation of the ladder operators for the rotating $\mathrm{G}-\mathrm{K}$ oscillator is the rotational-vibrational Schrödinger equation with the rotating $\mathrm{G}-\mathrm{K}$ potential [24]:

$$
\left\{-\frac{1}{2} \frac{d^{2}}{d r^{2}}+\frac{1}{2}\left[B^{\prime} r^{2}+\frac{A^{\prime}+J(J+1)}{r^{2}}\right]-\varepsilon_{v J}\right\} \Psi(r)_{v J}=0
$$

in which $\varepsilon_{v J}=\frac{2 m E_{v J}}{\hbar^{2}}$ and $\mathrm{J}=0,1,2 \ldots$ is the rotational quantum number.

The analytical solution of Eq. (3.1) has the following form [24]:

$$
\Psi(r)_{v J}=(-1)^{v}\left[\frac{2 \beta^{v}(\delta)_{v}}{v ! \Gamma(\delta)}\right]^{1 / 2} r^{\delta-\frac{1}{2}} \exp \left(-\frac{\beta r^{2}}{2}\right) F\left(-v, \delta, \beta r^{2}\right)
$$

in which

$$
\delta=1+\left[A^{\prime}+(J+1 / 2)^{2}\right]^{1 / 2} .
$$

Using the relation between the hypergeometric confluent function and associated Laguerre polynomial (2.8) we can rewrite the wave function (3.2) in the representation of a new variable $x=\beta r^{2}$ :

$$
\Psi(x)_{v J}=N_{v J} x^{\frac{1}{2}\left(\delta-\frac{1}{2}\right)} \exp \left(-\frac{x}{2}\right) L_{v}^{\delta-1}(x),
$$

in which

$$
N_{v J}=(-1)^{v}\left[\frac{2 \beta^{\delta}(\delta)_{v}}{v ! \Gamma(\delta)}\right]^{\frac{1}{2}} \beta^{-\frac{1}{2}\left(\delta-\frac{1}{2}\right)} \frac{v !(\delta-1) !}{(v+\delta-1) !}
$$


The ladder operators for the rotating G-K oscillator can be constructed directly from the wave function (3.4). To this aim, we are looking for the operators:

$$
\hat{L}_{+}=C_{+}(x) \frac{d}{d x}+D_{+}(x) \text { and } \hat{L}_{-}=C_{-}(x) \frac{d}{d x}+B_{-}(x) .
$$

which satisfy the following relationships:

$$
\hat{L}_{+} \Psi(x)_{v J}=L_{+}^{v J} \Psi(x)_{v+1, J} \text { and } \hat{L}_{-} \Psi(x)_{v J}=L_{-}^{v J} \Psi(x)_{v-1, J}
$$

Using Eq. (2.13) and the result of the action of the differential operator $\frac{d}{d x}$ on the wave function (3.4) one gets

$$
\frac{d}{d x} \Psi(x)_{v J}=\frac{\delta-\frac{1}{2}}{2 x} \Psi(x)_{v J}-\frac{1}{2} \Psi(x)_{v J}+N_{v J} x^{\frac{1}{2}\left(\delta-\frac{1}{2}\right)} \exp \left(-\frac{x}{2}\right) \frac{d}{d x} L_{v}^{\delta-1}(x) .
$$

After simple calculation, the lowering operator and its coefficient can be specified in the forms:

$$
\hat{L_{-}}=-\left[x \frac{d}{d x}-\frac{1}{2}\left(\delta-\frac{1}{2}\right)+\frac{1}{2} x-v\right], \quad L_{-}^{v J}=(v+\delta-1) \frac{N_{v J}}{N_{v-1, J}} .
$$

Additionally, one can show that this operator annihilates the vibrational ground state, which depends on the rotational quantum number:

$$
\hat{L_{-}} \Psi(x)_{0 J}=0
$$

In a similar manner one can construct the corresponding raising operator. Using Eq. (2.14) and the result of the action of the differential operator $\frac{d}{d x}$ on the wave function (3.4) one gets

$$
\frac{d}{d x} \Psi(x)_{v J}=\frac{\delta-\frac{1}{2}}{2 x} \Psi(x)_{v J}-\frac{1}{2} \Psi(x)_{v J}+N_{v J} \Phi(x)_{J}\left[\frac{v+1}{x} L_{v+1}^{\delta-1}(x)-\frac{v+\delta-x}{x} L_{v}^{\delta-1}(x)\right],
$$

in which

$$
\Phi(x)_{J}=x^{\frac{1}{2}\left(\delta-\frac{1}{2}\right)} \exp \left(-\frac{x}{2}\right) .
$$

In view of the above, the raising operator and its coefficient can be given in the forms:

$$
\hat{L_{+}}=x \frac{d}{d x}-\frac{1}{2}\left(\delta-\frac{1}{2}\right)-\frac{1}{2} x+v+\delta, \quad L_{+}^{v J}=(v+1) \frac{N_{v J}}{N_{v+1, J}} .
$$




\section{Coherent states of the G-K oscillator}

In this section we turn our attention towards the coherent states of the G-K potential. Crucial for the realization of this aim is an assumption that the Hamiltonian can be factorized as

$$
\hat{H}_{0}=\hat{A}^{\dagger} \hat{A}+E_{0},
$$

in which

$$
\hat{A}^{\dagger}=\frac{1}{\sqrt{2}}\left[-\frac{d}{d r}+W_{0}(r)\right], \quad \hat{A}=\frac{1}{\sqrt{2}}\left[\frac{d}{d r}+W_{0}(r)\right], \quad\left[\hat{A}, \hat{A^{\dagger}}\right]=-\frac{d W_{0}(r)}{d r} .
$$

Here $\mathrm{W}_{0}(\mathrm{r})$ is interpreted as a Witten superpotential [35,36], which satisfies the wellknow Riccati differential equation:

$$
\frac{\hbar^{2}}{2 m}\left[W_{0}^{2}(r)-\frac{d W_{0}(r)}{d r}\right]+V(r)=E_{0},
$$

which is widely used in sypersymmetric quantum mechanics (SUSY QM) and permits a construction of the Schrödinger equation which has analytical solutions [37]. The explicit expression for the superpotential can be determined using the Darboux transformation. Hence, employing this approach, $W_{0}(r)$ can be specified by a general formula:

$$
W_{0}(r)=-\frac{d}{d r} \ln \Psi(r)_{0 J},
$$

in which $\Psi(r)_{0 J}$ denotes the vibrational ground state of the quantum system. Taking into account the wave function (3.2) in the arbitrary normalization one obtains [37]

$$
W_{0}(r)=\frac{-\delta+\frac{1}{2}}{r}+\beta r
$$

Equations (4.2) and (4.5) generate the annihilation and creation operators for the G-K potential:

$$
\hat{A^{\dagger}}=\frac{1}{\sqrt{2}}\left[-\frac{d}{d r}+\frac{-\delta+\frac{1}{2}}{r}+\beta r\right], \quad \hat{A}=\frac{1}{\sqrt{2}}\left[\frac{d}{d r}+\frac{-\delta+\frac{1}{2}}{r}+\beta r\right] .
$$

Additionally, it can be shown that the operator $\hat{A}$ annihilates the ground state. Therefore, the coherent state $|\alpha J\rangle$ of the rotating $\mathrm{G}-\mathrm{K}$ oscillator is the eigenstate of the annihilation operator:

$$
\hat{A}|\alpha J\rangle=\alpha|\alpha J\rangle, \quad|\alpha J\rangle=r^{\delta-\frac{1}{2}} \exp \left(-\frac{\beta r^{2}}{2}+\sqrt{2} \alpha r\right)
$$


The method proposed also permits a derivation of the coherent states for the $\mathrm{G}-\mathrm{K}$ oscillator with zero total angular momentum. Applying this procedure, after similar calculations, the normalized coherent states can be given in the form:

$$
|\alpha\rangle=N_{\alpha} r^{\gamma-\frac{1}{2}} \exp \left(-\frac{\beta r^{2}}{2}+\sqrt{2} \alpha r\right)
$$

It is interesting to note that the isospectral partner potential $V_{D}(r)$ (Darboux potential) associated with the three-dimensional rotating $\mathrm{G}-\mathrm{K}$ potential satisfies the following equation:

$$
V_{D}(r)=V(r)-2 \frac{d^{2}}{d r^{2}} \Psi(r)_{0 J}
$$

Using Eq. (4.5) one gets the Darboux potential:

$$
V_{D}(r)=V(r)+2\left(\frac{\delta-\frac{1}{2}}{r^{2}}+\beta\right) .
$$

The ground state solution associated with the standard Darboux potential can be generated from the following relationship [14]:

$$
\psi(r)_{0 J}=\exp \left[-\int W_{0}(r) d r\right]
$$

Hence, the ground state wave function can be specified by a general formula:

$$
\psi(r)_{0 J}=r^{\delta-1 / 2} \exp \left(-\frac{\beta r^{2}}{2}\right)
$$

\section{Minimum uncertainty coherent states}

It is easy to demonstrate that the coherent states $|\alpha J\rangle$ minimize the generalized position-momentum uncertainty relation $(\hbar=1)[14]$ :

$$
[\Delta y(r)]^{2}(\Delta p)^{2} \geq \frac{1}{4}\langle\alpha J|g(r)| \alpha J\rangle^{2}, \quad[y(r), \hat{p}]=i g(r)=i \frac{d y(r)}{d r}=-\left[\hat{A}, \hat{A}^{\dagger}\right],
$$

in which $\mathrm{y}(r)$ denotes an anharmonic coordinate, whose explicit form depends on the oscillator type, whereas $\hat{p}=-i \frac{d}{d r}$ is the associated momentum operator. Adopting Eq. (5.1) to the rotating $\mathrm{G}-\mathrm{K}$ potential we obtain

$$
y(r)=\frac{-\delta+\frac{1}{2}}{r}+\beta r .
$$


Taking into account Eqs. (5.1) and (5.2) one gets

$$
g(r)=\frac{\delta-\frac{1}{2}}{r^{2}}+\beta
$$

In the next step we can calculate the matrix elements:

$$
\begin{aligned}
\langle\alpha J|y(r)| \alpha J\rangle & =\frac{1}{\sqrt{2}}\left\langle\alpha J\left|\hat{A}+\hat{A}^{\dagger}\right| \alpha J\right\rangle=\frac{1}{\sqrt{2}}\left(\alpha+\alpha^{*}\right), \\
\langle\alpha J|\hat{p}| \alpha J\rangle & =-i \frac{1}{\sqrt{2}}\left\langle\alpha J\left|\hat{A}-\hat{A}^{\dagger}\right| \alpha J\right\rangle=-i \frac{1}{\sqrt{2}}\left(\alpha-\alpha^{*}\right), \\
2\left\langle\alpha J\left|y^{2}(r)\right| \alpha J\right\rangle & =\left\langle\alpha J\left|\hat{A} \hat{A}+2 \hat{A}^{\dagger} \hat{A}+\hat{A}^{\dagger} \hat{A}^{\dagger}-g(r)\right| \alpha J\right\rangle=\left(\alpha-\alpha^{*}\right)^{2}-\langle\alpha J|g(r)| \alpha J\rangle, \\
-2\left\langle\alpha J\left|\hat{p}^{2}\right| \alpha J\right\rangle & =\left\langle\alpha J\left|\hat{A} \hat{A}-2 \hat{A}^{\dagger} \hat{A}+\hat{A}^{\dagger} \hat{A}^{\dagger}+g(r)\right| \alpha J\right\rangle=\left(\alpha-\alpha^{*}\right)^{2}+\langle\alpha J|g(r)| \alpha J\rangle,
\end{aligned}
$$

applying the following operator relation:

$$
\hat{A} \hat{A}^{\dagger}=\hat{A}^{\dagger} \hat{A}-g(r)
$$

Taking into account Eqs. (5.4)-(5.7) one may evaluate

$$
\begin{aligned}
{[\Delta y(r)]^{2} } & =\left\langle\alpha J\left|y(r)^{2}\right| \alpha J\right\rangle-\langle\alpha J|y(r)| \alpha J\rangle=-\frac{1}{2}\langle\alpha J|g(r)| \alpha J\rangle, \\
(\Delta p)^{2} & =\left\langle\alpha J\left|\hat{p}^{2}\right| \alpha J\right\rangle-\langle\alpha J|\hat{p}| \alpha J\rangle-\frac{1}{2}\langle\alpha J|g(r)| \alpha J\rangle,
\end{aligned}
$$

providing that $\Delta y(r)=\Delta p$ and

$$
[\Delta y(r)]^{2}(\Delta p)^{2}=\frac{1}{4}\langle\alpha J|g(r)| \alpha J\rangle^{2}=\frac{1}{4}\left\langle\alpha J\left|\frac{\delta-\frac{1}{2}}{r^{2}}+\beta\right| \alpha J\right\rangle^{2}
$$

The simple calculations performed reveal that the obtained coherent states minimize the generalized position-momentum uncertainty relation for the $\mathrm{G}-\mathrm{K}$ anharmonic coordinate $\mathrm{y}(r)$ and that they are the eigenstates of the operator $\hat{A}$, which annihilates the vibrational ground state $\hat{A}|0 J\rangle=0$. Therefore, they satisfy the two fundamental requirements established for the coherent states of an anharmonic oscillator. Some similar minimum-uncertainty coherent states have been recently derived algebraically by Molski [38] and Mikulski et al. [39]. 
The integral on the right-hand side of the relation (5.11) can be calculated using the well-known formula:

$$
\int_{0}^{\infty} r^{n} e^{-\mu r} d r=n ! \mu^{-(n+1)}, \quad \operatorname{Re}(\mu)>0
$$

and the integral representation of the Laguerre function. The starting point for the realization of this aim is the calculation of the normalization constant of $|\alpha J\rangle$ (4.7):

$$
|\alpha J\rangle=r^{\delta-\frac{1}{2}} \exp \left(-\frac{\beta r^{2}}{2}+\sqrt{2} \alpha r\right) .
$$

After simple calculations we obtain the normalization constant in the following form:

$$
\begin{aligned}
N_{\alpha J}= & \langle\alpha J \mid \alpha J\rangle^{-1 / 2}=\left(\frac{\beta^{-\delta}}{2 \sqrt{\pi}}\right)^{1 / 2}\left\{\frac{\pi^{2}\left[\frac{\left(\alpha+\alpha^{*}\right)^{2}}{\beta}+1\right] L\left[-\delta, \frac{1}{2}, \frac{\left(\alpha+\alpha^{*}\right)^{2}}{2 \beta}\right]}{2 \sin (\delta \pi) \Gamma\left(\frac{3}{2}-\delta\right)}\right. \\
& -\frac{\pi^{2}\left(\alpha+\alpha^{*}\right)^{2} L\left[-\delta, \frac{3}{2}, \frac{\left(\alpha+\alpha^{*}\right)^{2}}{2 \beta}\right]}{2 \beta \sin (\delta \pi) \Gamma\left(\frac{3}{2}-\delta\right)} \\
& +\frac{\sqrt{2} \pi^{2}\left(\alpha+\alpha^{*}\right)\left[\frac{\left(\alpha+\alpha^{*}\right)^{2}}{\beta}+2 \delta-1\right] L\left[-\delta+\frac{1}{2}, \frac{1}{2}, \frac{\left(\alpha+\alpha^{*}\right)^{2}}{2 \beta}\right]\left(-\delta+\frac{1}{2}\right)}{2 \sqrt{\beta}(2 \delta-1) \cos (\delta \pi) \Gamma(2-\delta)} \\
& \left.-\frac{\sqrt{2} \pi^{2}\left(\alpha+\alpha^{*}\right)^{3} L\left[-\delta+\frac{1}{2}, \frac{3}{2}, \frac{\left(\alpha+\alpha^{*}\right)^{2}}{2 \beta}\right]\left(-\delta+\frac{1}{2}\right)}{2 \beta^{3 / 2}(2 \delta-1) \cos (\delta \pi) \Gamma(2-\delta)}\right\}^{1 / 2},
\end{aligned}
$$

in which $L(a, b, x)$ denotes the Laguerre function. In this way we obtain the following result:

$$
\begin{aligned}
& \left\langle\alpha J\left|\frac{\delta-\frac{1}{2}}{r^{2}}+\beta\right| \alpha J\right\rangle=\frac{N_{\alpha J}^{2} 2^{\delta-\frac{5}{2}} \Omega\left(-\alpha-\alpha^{*}\right)^{-2 \delta+3}}{\sqrt{\pi \beta}} \\
& \quad \times\left\{\frac{2^{-\delta+1} \pi^{2}\left(-\alpha-\alpha^{*}\right)^{2 \delta-2} \beta^{-\delta+1} L\left[-\delta+\frac{1}{2}, \frac{1}{2}, \frac{\left(\alpha+\alpha^{*}\right)^{2}}{2 \beta}\right]}{\cos (\delta \pi) \Gamma(2-\delta)}\right.
\end{aligned}
$$




$$
\begin{aligned}
& -\frac{2^{-\delta+1 / 2} \pi^{2}\left(-\alpha-\alpha^{*}\right)^{2 \delta-3} \beta^{3 / 2-\delta} L\left[-\delta, \frac{1}{2}, \frac{\left(\alpha+\alpha^{*}\right)^{2}}{2 \beta}\right]}{\sin (\delta \pi) \Gamma\left(\frac{3}{2}-\delta\right)(-\delta+1)} \\
& \left.+\frac{2^{-\delta+1 / 2} \pi^{2}\left(-\alpha-\alpha^{*}\right)^{2 \delta-1} \beta^{-\delta+1 / 2} L\left[-\delta, \frac{3}{2}, \frac{\left(\alpha+\alpha^{*}\right)^{2}}{2 \beta}\right]}{\sin (\delta \pi) \Gamma\left(\frac{3}{2}-\delta\right)(-\delta+1)}\right\}+\beta,
\end{aligned}
$$

in which

$$
\Omega=\delta-\frac{1}{2}
$$

Basing on the result (5.11) we can finally write

$$
\begin{aligned}
& \Delta y(r) \Delta p \\
& =\frac{N_{\alpha J}^{2} 2^{\delta-\frac{5}{2}} \Omega\left(-\alpha-\alpha^{*}\right)^{-2 \delta+3}}{2 \sqrt{\pi \beta}} \\
& \quad \times\left\{\frac{2^{-\delta+1} \pi^{2}\left(-\alpha-\alpha^{*}\right)^{2 \delta-2} \beta^{-\delta+1} L\left[-\delta+\frac{1}{2}, \frac{1}{2}, \frac{\left(\alpha+\alpha^{*}\right)^{2}}{2 \beta}\right]}{\cos (\delta \pi) \Gamma(2-\delta)}\right. \\
& \left.-\frac{2^{-\delta+1 / 2} \pi^{2}\left(-\alpha-\alpha^{*}\right)^{2 \delta-3} \beta^{3 / 2-\delta} L\left[-\delta, \frac{1}{2}, \frac{\left(\alpha+\alpha^{*}\right)^{2}}{2 \beta}\right]}{\sin (\delta \pi) \Gamma\left(\frac{3}{2}-\delta\right)(-\delta+1)}\right] \\
& \left.+\frac{2^{-\delta+1 / 2} \pi^{2}\left(-\alpha-\alpha^{*}\right)^{2 \delta-1} \beta^{-\delta+1 / 2} L\left[-\delta, \frac{3}{2}, \frac{\left(\alpha+\alpha^{*}\right)^{2}}{2 \beta}\right]}{\sin (\delta \pi) \Gamma\left(\frac{3}{2}-\delta\right)(-\delta+1)}\right]+\frac{1}{2} \beta .
\end{aligned}
$$

It should be also pointed out that the states $|\alpha\rangle$ minimize the generalized positionmomentum uncertainty relation (5.1) yielding

$$
[\Delta y(r)]^{2}(\Delta p)^{2}=\frac{1}{4}\left\langle\alpha\left|\frac{\gamma-\frac{1}{2}}{r^{2}}+\beta\right| \alpha\right\rangle^{2},
$$

where

$$
y(r)=\frac{-\gamma+\frac{1}{2}}{r}+\beta r .
$$

In Eq. (5.18) $y(r)$ is the $\mathrm{G}-\mathrm{K}$ coordinate, whereas $\frac{(\gamma-1 / 2)}{r^{2}}-\beta=g(r)$.

Proceeding along the lines of the previous calculation one may solve the integral on the right-hand side of the relation (5.18) yielding 


$$
\begin{aligned}
& \Delta y(r) \Delta p=\frac{N_{\alpha}^{2} 2^{\gamma-\frac{5}{2}}\left(\gamma-\frac{1}{2}\right)\left(-\alpha-\alpha^{*}\right)^{-2 \gamma+3}}{2 \sqrt{\pi \beta}} \\
& \quad \times\left\{\frac{2^{-\gamma+1} \pi^{2}\left(-\alpha-\alpha^{*}\right)^{2 \gamma-2} \beta^{-\gamma+1} L\left[-\gamma+\frac{1}{2}, \frac{1}{2}, \frac{\left(\alpha+\alpha^{*}\right)^{2}}{2 \beta}\right]}{\cos (\gamma \pi) \Gamma(2-\gamma)}\right. \\
& \left.-\frac{2^{-\gamma+1 / 2} \pi^{2}\left(-\alpha-\alpha^{*}\right)^{2 \delta-3} \beta^{3 / 2-\gamma} L\left[-\gamma, \frac{1}{2}, \frac{\left(\alpha+\alpha^{*}\right)^{2}}{2 \beta}\right]}{\sin (\gamma \pi) \Gamma\left(\frac{3}{2}-\gamma\right)(-\gamma+1)}\right]+\frac{1}{2} \beta, \\
& \left.+\frac{2^{-\gamma+1 / 2} \pi^{2}\left(-\alpha-\alpha^{*}\right)^{2 \gamma-1} \beta^{-\gamma+1 / 2} L\left[-\gamma, \frac{3}{2}, \frac{\left(\alpha+\alpha^{*}\right)^{2}}{2 \beta}\right]}{\sin (\gamma \pi) \Gamma\left(\frac{3}{2}-\gamma\right)(-\gamma+1)}\right]
\end{aligned}
$$

in which

$$
\begin{aligned}
N_{\alpha}=\langle\alpha \mid \alpha\rangle^{-1 / 2} & \\
= & \left(\frac{\beta^{-\gamma}}{2 \sqrt{\pi}}\right)^{1 / 2}\left\{\frac{\pi^{2}\left[\frac{\left(\alpha+\alpha^{*}\right)^{2}}{\beta}+1\right] L\left[-\gamma, \frac{1}{2}, \frac{\left(\alpha+\alpha^{*}\right)^{2}}{2 \beta}\right]}{2 \sin (\gamma \pi) \Gamma\left(\frac{3}{2}-\gamma\right)}\right. \\
& -\frac{\pi^{2}\left(\alpha+\alpha^{*}\right)^{2} L\left[-\gamma, \frac{3}{2}, \frac{\left(\alpha+\alpha^{*}\right)^{2}}{2 \beta}\right]}{2 \beta \sin (\gamma \pi) \Gamma\left(\frac{3}{2}-\gamma\right)} \\
& +\frac{\sqrt{2} \pi^{2}\left(\alpha+\alpha^{*}\right)\left[\frac{\left(\alpha+\alpha^{*}\right)^{2}}{\beta}+2 \gamma-1\right] L\left[-\gamma+\frac{1}{2}, \frac{1}{2}, \frac{\left(\alpha+\alpha^{*}\right)^{2}}{2 \beta}\right]\left(-\gamma+\frac{1}{2}\right)}{2 \sqrt{\beta}(2 \gamma-1) \cos (\gamma \pi) \Gamma(2-\gamma)} \\
& \left.-\frac{\sqrt{2} \pi^{2}\left(\alpha+\alpha^{*}\right)^{3} L\left[-\gamma+\frac{1}{2}, \frac{3}{2}, \frac{\left(\alpha+\alpha^{*}\right)^{2}}{2 \beta}\right]\left(-\gamma+\frac{1}{2}\right)}{2 \beta^{3 / 2}(2 \gamma-1) \cos (\gamma \pi) \Gamma(2-\gamma)}\right\}^{1 / 2} .
\end{aligned}
$$

In the next study we will derive the dynamical symmetry group to show that these ladder operators satisfy SU(1,1) Lie group. Moreover, in the next paper we will show relevant applications of the ladder operators and coherent states derived in quantum chemistry computations. In particular, we will analytically compute the matrix elements for the vibrational and rotational-vibrational transitions in several diatomic molecules.

\section{Conclusions}

In this work the ladder operators for the $\mathrm{G}-\mathrm{K}$ potential have been constructed by a simple algebraic procedure that had been applied by Dong et al. [18] to the Morse potential. These operators have been obtained directly from the analytical eigenfunc- 
tion of the Schrödinger equation for the $\mathrm{G}-\mathrm{K}$ oscillator. Also in this study the coherent states of the G-K potential and the associated Darboux potential have been constructed using the SUSY quantum mechanics method. It has been proved that these states minimize the generalized position-momentum uncertainty relation. We hope that the ladder operators constructed will be applied by other researchers to compute transition matrix elements in theoretical spectroscopy of diatomic molecules.

Open Access This article is distributed under the terms of the Creative Commons Attribution License which permits any use, distribution, and reproduction in any medium, provided the original author(s) and the source are credited.

\section{References}

1. W.M. Zhang, D.M. Feng, R. Gilimore, Rev. Mod. Phys. 62, 867 (1990)

2. M.M. Nieto, L.M. Simmons, Phys. Rev. Lett. 41, 207 (1978)

3. S. Kais, R.D. Levine, Phys. Rev. A 41, 1671 (1990)

4. M.G. Benedict, B. Molnar, Phys. Rev. A 60, R1371 (1999)

5. T. Fukui, A. Aizawa, Phys. Lett. A 180, 308 (1993)

6. C.C. Gerry, Phys. Rev. A 33, 2207 (1986)

7. R.D. Levine, Chem. Phys. Lett. 95,87 (1983)

8. S. Kais, R.D. Levine, Phys. Rev A. 34, 4615 (1986)

9. M.G.A. Crawfor, E. Vrscay, Phys. Rev. A 57, 106 (1998)

10. A. Chenaghou, H. Fakhri, Mod. Phys. Lett. A 17, 1701 (2002)

11. C.C. Gerry, J. Kiefer, Phys. Rev. A 37, 665 (1988)

12. M.M. Nieto, L.M. Simmons, Phys. Rev. D 20, 1332 (1979)

13. P. Majumdar, H.S. Sharatchandra, Phys. Rev. A 56, R3322 (1997)

14. I.L. Cooper, J. Phys. A Math. Theor. 25, 1671 (1992)

15. A. Gangopadhyaya, K.P. Panigrahi, U.P. Sukhatme, J. Phys. A Math. Theor. 27, 4295 (1994)

16. E. Schrödinger, Proc. R. Ir. Acad. A 46(9), 183 (1941)

17. L. Infeld, T.E. Hull, Rev. Mod. Phys. 23, 21 (1951)

18. S.H. Dong, R. Lemus, A. Franc, Int. J. Quantum Chem. 83, 433 (2002)

19. S.H. Dong, R. Lemus, Int. J. Quantum Chem. 86, 265 (2002)

20. S.H. Dong, Appl. Math. Lett. 16, 199 (2003)

21. R. Gilmore, Lie Groups, Physics and Geometry, 1st edn. (Cambridge University Press, Cambridge, 2008)

22. N.M. Avram, G.E. Drặgặnescu, Int. J. Quantum Chem. 64, 655 (1997)

23. S.H. Dong, G.H. Sun, M. Lozada-Cassou, Phys. Lett. 340, 94 (2005)

24. R. Hall, N. Saad, J. Math. Gen. 31, 963 (1988)

25. A.M. Perelomov, Commun. Math. Phys. 26, 222 (1972)

26. S.H. Dong, Can. J. Phys. 80, 129 (2002)

27. M.M. Nieto, L.M. Simmons, Phys. Rev. A 19, 10 (1979)

28. A.O. Borut, L. Girardello, Commun. Math. Gen. 21, 41 (1971)

29. C. Brief, Int. J. Theor. Phys. 36, 1651 (1971)

30. H. Fakhri, A. Chenaghlou, Phys. Lett. A 310, 1 (2003)

31. J.R. Klauder, J. Math. Phys. 4, 1058 (1963)

32. J.P. Gazeau, J.R. Klauder, J. Phys. A 32, 123 (1999)

33. D. Popov, Phys. Lett. A 316, 369 (2003)

34. I.S. Gradshteyn, I.M. Ryzhik, Tables of Integrals Series and Products, 5 th edn. (Academic Press, San Diego, CA, 1994)

35. E. Witten, Nucl. Phys. B 188, 513 (1981)

36. J.J. Peña, M.A. Romero-Romo, J. Morales, J.L. López-Bonilla, Int. J. Quantum Chem. 105, 731 (2005)

37. F. Cooper, A. Khare, U. Sukhatme, Phys. Rep. 251, 267 (1995)

38. M. Molski, J. Phys. A Math. Theor. 42, 165301 (2009)

39. D. Mikulski, M. Molski, K. Eder, J. Math. Chem. (2013). doi:10.1007/s10910-013-0251-7 